\title{
EFFECTS OF INCOHERENT SPACE CHARGE FORCES ON THE FAST HEAD-TAIL INSTABILITY
}

\section{Abstract}

The effects of incoherent space charge forces on the fast head-tail instability are studied numerically. It is found that incoherent space charge forces can dramatically increase the threshold current for a fixed wall impedance.

\section{PHYSICAL MODEL AND SIMULATION ALGORITHM}

For the purposes of beam dynamics one transverse and the longitudinal degrees of freedom are considered. Let $\theta$ denote the machine azimuth, which increases by $2 \pi$ each turn and will be used as the time-like variable. The time is denoted by $t$ and $\omega_{0}$ is the angular revolution frequency of a synchronous particle. Consider a single particle and let $\tau(\theta)=t-\theta / \omega_{0}$ denote the time delay between this particle reaching $\theta$ and the synchronous particle reaching $\theta$. The longitudinal equation of motion for a single particle is approximated as

$$
\frac{d^{2} \tau}{d^{2} \theta}+Q_{s}^{2} \tau=0
$$

where $Q_{s}$ is the synchrotron tune. The single particle equation of motion for the transverse degree of freedom $x$ is approximated as

$$
\begin{gathered}
\frac{d^{2} x}{d^{2} \theta}=-Q_{x}^{2} x+C_{s c} \rho(\theta, \tau)(x-\langle x(\theta, \tau)\rangle) \\
+\int_{-T}^{\tau} d \tau^{\prime} W\left(\tau-\tau^{\prime}\right) \rho\left(\theta, \tau^{\prime}\right)\left\langle x\left(\theta, \tau^{\prime}\right)\right\rangle .
\end{gathered}
$$

In equation (2) $Q_{x}$ is the bare betatron, $C_{s c} \geq 0$ characterizes the peak strength of the incoherent space charge force, and $\rho(\theta, \tau)$ is the line density of the particles which vanishes for $|\tau|>T$, the half length of the bunch. The transverse center of the beam as a function of azimuth and delay is $\langle x(\theta, \tau)\rangle$, and the causal coherent forces due to wall impedances are characterized by the wake potential $W(\tau)$. Extending the model to include chromaticity and long range wake forces is straightforward, but will not be considered here.

Equations (1) and (2) are solved by particle tracking. The bunch is modeled as $N$ interacting macro-particles. The equations of motion for $k$ th macro-particle are taken to be

$$
\frac{d^{2} \tau_{k}}{d^{2} \theta}=-Q_{s}^{2} \tau_{k}
$$

\footnotetext{
- Work supported by United States Department of Energy
}

$$
\begin{aligned}
\frac{d^{2} x_{k}}{d^{2} \theta} & =-Q_{x}^{2} x_{k}+\frac{C_{s c}}{N} \sum_{j=1}^{N}\left(x_{k}-x_{j}\right) \lambda\left(\tau_{k}-\tau_{j}\right) \\
& +\frac{1}{N} \sum_{j=1}^{N} x_{j} \hat{W}\left(\tau_{k}-\tau_{j}\right) .
\end{aligned}
$$

In equation 4 the new functions $\lambda(\tau)$ and $\hat{W}(\tau)$ are introduced to smooth out the particle-particle forces. If one takes the limit $N \rightarrow \infty$ and then takes the limits of $\lambda(\tau)$ going to a delta function and $\hat{W}(\tau) \rightarrow W(\tau)$, equation (2) is recovered.

The number of macro-particles is controlled using the parameter $n_{\ell}$. The initial longitudinal variables, $\tau_{k}$, and $v_{k}=d \tau_{k} /(Q, d \theta)$, are selected by considering the subset of lattice points $\left(T_{k}, V_{k}\right)=\left(\left(k_{\tau}+1 / 2\right) / n_{\ell},\left(k_{v}+\right.\right.$ $\left.1 / 2) / n_{l}\right)$, with $k_{\tau}$ and $k_{v}$ integers, which are inside the unit circle. The initial longitudinal coordinates of a macro particle are derived via

$$
\left(\tau_{k}, v_{k}\right)=L\left(T_{k}, V_{k}\right)\left(1-\left(1-R_{k}^{2}\right)^{1 /(1+\mu)}\right)^{1 / 2} / R_{k},
$$

where the parameter $\mu$ determines the bunch shape and $R_{k}^{2}=T_{k}^{2}+V_{k}^{2}$. The smoothed density in longitudinal phase space is proportional to $\left(L^{2}-\tau^{2}-v^{2}\right)^{\mu}$, and results in a line density $\propto\left(L^{2}-\tau^{2}\right)^{\mu+1 / 2}$. During the simulation the longitudinal variables were updated once per turn using a rotation with angle $2 \pi Q_{s}$. Figure 1 illustrates the selection of longitudinal coordinates.

The initial values of the transverse variables $x_{k}$ and $p_{k}=d x_{k} / d \theta$ were obtained using a random number generator. The transverse dynamics consists of two parts, single particle dynamics and multiparticle dynamics. The scheme involves a single particle update followed by a multiparticle update and is repeated $M$ times per turn. The application of collective forces once per turn, as is usually done in lepton machines [1], is not sufficient since space charge tune shifts are large. The single particle update is given by a transfer matrix with a bare betatron phase advance $2 \pi Q_{x} / M$. The multiparticle update consists of a kick from the space charge and wake forces. The space charge kick is given by

$$
F_{k}=\hat{C}_{s c} \sum_{j=1}^{N}\left(x_{k}-x_{j}\right) \lambda\left(\tau_{k}-\tau_{j}\right),
$$

where $\hat{C}_{s c}=2 \pi C_{s c} / N M$. The kick due to the wake potential is

$$
F_{k}=\frac{2 \pi}{N M} \sum_{j=1}^{N} x_{j} \hat{W}\left(\tau_{k}-\tau_{j}\right) .
$$




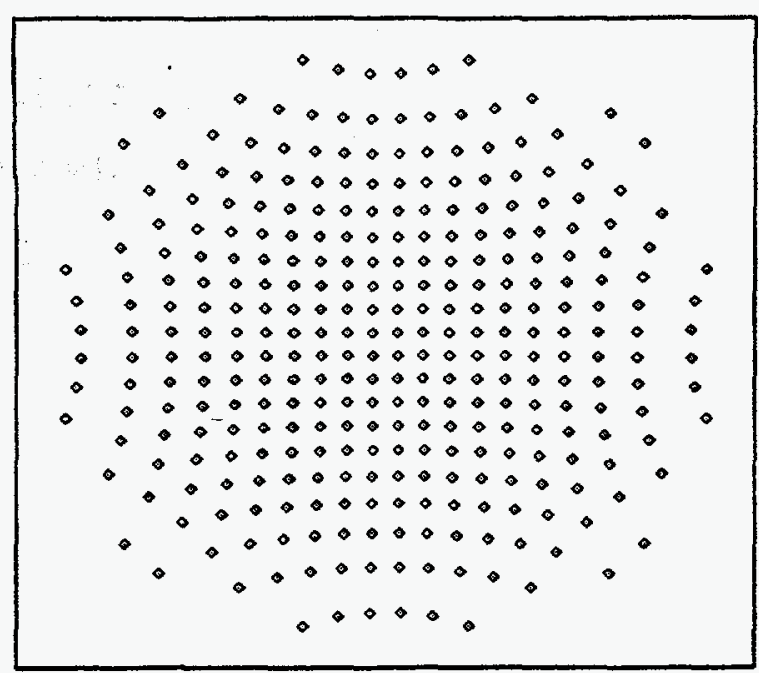

Figure 1: Initial longitudinal coordinates for $n_{\ell}=10$ and $\mu=1$. There are $316 \approx \pi n_{l}^{2}$ macro-particles.

On the surface, equations (5) and (6) appear to require $O\left(N^{2}\right)$ operations to obtain the kicks for all $N$ macroparticles. This would make simulations with large $N$ untenable. For appropriate choices of $\lambda(\tau)$ and $\hat{W}(\tau)$ the operation count drops to $O(N \log (N))$. The trick is to generalize the phasor technique which is usually employed to retain the cumulative effects of multiple passages through a resonant structure[2].

The smoothing functions

$$
\lambda(\tau)=\exp (-\alpha|\tau|),
$$

where $\int \lambda d \tau=2 / \alpha=\tau_{e}$ is the equivalent duration of $\lambda(\tau)$, and

$$
\lambda_{2}(\tau)=(1+\alpha|\tau|) \exp (-\alpha|\tau|)
$$

with $4 / \alpha=\tau_{e}$ can be summed efficiently. The algorithm for $\lambda_{2}(\tau)$ is an obvious generalization of the algorithm for $\lambda(\tau)$, which is presented in detail.

Sort the values of $\tau_{j}$ so that $\tau_{j} \leq \tau_{j+1}$, which is an $O(N \log N)$ process with standard algorithms[3]. Equation (5) is given by

$$
F_{k} / \hat{C}_{s c}=x_{k} S 1_{k}^{-}-S 2_{k}^{-}+x_{k} S 1_{k}^{+}-S 2_{k}^{+},
$$

where

$$
\begin{aligned}
& S 1_{k}^{-}=\sum_{j=1}^{k} e^{\alpha\left(\tau_{j}-\tau_{k}\right)}, \\
& S 2_{k}^{-}=\sum_{j=1}^{k} x_{j} e^{\alpha\left(\tau_{j}-\tau_{k}\right)}, \\
& S 1_{k}^{+}=\sum_{j=k+1}^{N} e^{\alpha\left(\tau_{k}-\tau_{j}\right)},
\end{aligned}
$$

$$
S 2_{k}^{+}=\sum_{j=k+1}^{N} x_{j} e^{\alpha\left(\tau_{k}-\tau_{j}\right)} .
$$

To calculate the sums one starts with $S_{N}^{+}=0, S_{0}^{-}=0$ and uses

$$
\begin{aligned}
& S 1_{k+1}^{-}=e^{\alpha\left(\tau_{k}-\tau_{k+1}\right)} S 1_{k}^{-}+1, \\
& S 2_{k+1}^{-}=e^{\alpha\left(\tau_{k}-\tau_{k+1}\right)} S 2_{k}^{-}+x_{k+1}, \\
& S 1_{k-1}^{+}=e^{\alpha\left(\tau_{k-1}-\tau_{k}\right)}\left(S 1_{k}^{+}+1\right), \\
& S 2_{k-1}^{+}=e^{\alpha\left(\tau_{k-1}-\tau_{k}\right)}\left(S 2_{k}^{+}+x_{k}\right),
\end{aligned}
$$

Note that these recurrence relations are stable and that the kicks for all $N$ particles require $O(N)$ calculations after the particles have been sorted in arrival time. The sorting procedure is done when the $\tau_{k} s$ are updated, once per turn.

Next, consider the kick due to the transverse wake field. A smoothed version of the step function wake given by $W(\tau)=W$ for $\tau>0$ and $W(\tau)=0$ for $\tau<0$ is

$$
\hat{W}(\tau)=C_{W} \int_{-\infty}^{\tau} \lambda\left(\tau^{\prime}\right) d \tau^{\prime}
$$

where $C_{W}$ is a constant and $\lambda(t)=\exp (-\alpha|\tau|)$, as before. Adjusting the constant so that $\hat{W}(\tau) \rightarrow W$ as $\tau \rightarrow \infty$ yields

$$
F_{k}=\frac{2 \pi W}{N M}\left[S 0_{k}^{-}-S 2_{k}^{-} / 2+S 2_{k}^{+} / 2\right],
$$

where

$$
S 0_{k}^{-}=\sum_{j=1}^{k} x_{j} .
$$

For a resonator impedance with resonant frequency $\omega_{r}$ and quality factor $Q_{r}$ the wake kick on particle $k$ is given by

$$
F_{k}=\sum_{j=1}^{k} x_{j} W_{r} \sin \left[\tilde{\omega}\left(\tau_{k}-\tau_{j}\right)\right] e^{-\omega_{r}\left(\tau_{k}-\tau_{j}\right) / 2 Q_{r}}
$$

where $\tilde{\omega}=\omega_{r} \sqrt{1-1 / 4 Q_{r}^{2}}$. Using $\exp (i \phi)=\cos (\phi)+$ $i \sin (\phi)$ a complex sum similar to $S 2_{k}^{-}$is obtained.

\section{SIMULATION RESULTS}

The algorithm described in the previous section has been implemented in fortran code. Results with the smoothing function $\lambda_{2}(\tau)$ and the step function wake will be presented. Values of $n_{\ell}, \mu, Q_{s}, \tau_{e}, Q_{x}, M, W$, and the peak value of the incoherent space charge tune shift $\Delta Q_{s c}$ were chosen. For the results presented here $M=24, Q_{x}=2.9$, and $Q_{s}=0.01 \rightarrow 0.1$.

The simulations were allowed to continue until the betatron oscillation showed a clear exponential growth rate. The growth rate of the exponential was identified as the growth rate of the most unstable mode. The values of the 


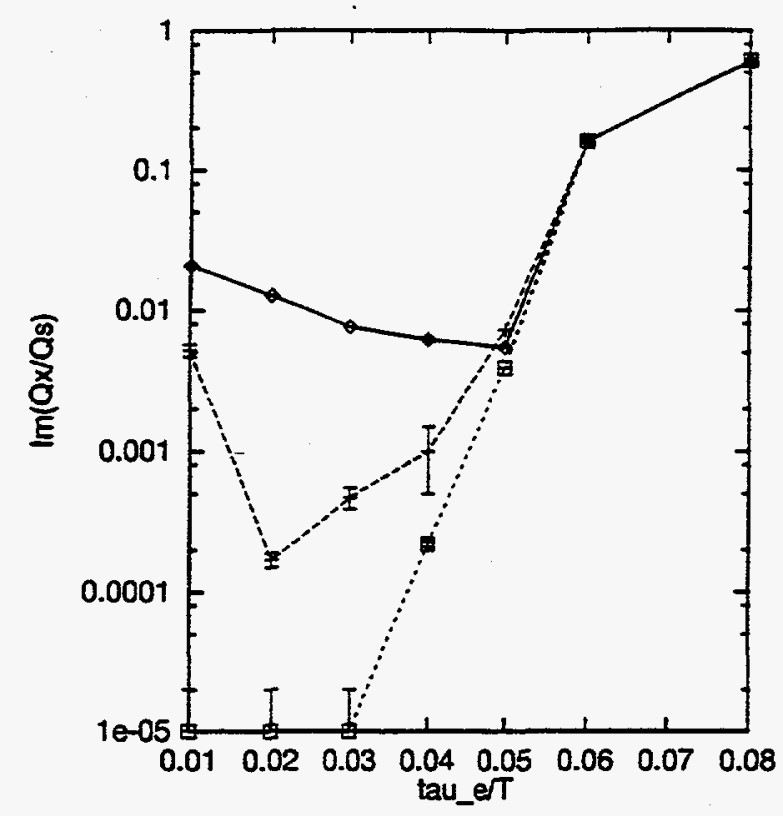

Figure 2: $\operatorname{Im}\left(Q_{x} / Q_{s}\right)$ versus $\tau_{e} / T$ with $\mu=1$ and $\Delta Q_{s c} / Q_{s}=20$ for various values of $n_{\ell}: n_{\ell}=25$, solid line; $n_{\ell}=50$, long dash; $n_{\ell}=100$, short dash. The value of the step function wake was five times larger than the threshold value with $\Delta Q_{s c}=0$.

growth rate were insensitive to the seed value of the random number generator and were the same in both single and double precision.

Figure 2 shows the growth rate of the most unstable mode as a function of $\tau_{e} / T$ for a system which would be highly unstable in the absence of space charge forces. From the figure the best values of $\tau_{e}$ were $0.05,0.02$ and 0.01 for $n_{\ell}=25,50$, and 100 , respectively.

Figure 3 shows $\operatorname{Im}\left(Q_{x} / Q_{s}\right)$ versus $\Delta Q_{s c} / q s$. While there is a noticeable rise in growth rate after the initial decay $\operatorname{Im}\left(Q_{x} / Q_{s}\right)$ stays below $1 \%$ of its value in the absence of space charge. A threshold value of $\Delta Q_{s c} / Q_{s}=2$ is inferred from this plot

Figure 4 is the main result. The threshold value of the wake potential increases with space charge tune shift. Results for smooth $(\mu=1)$ and boxcar $(\mu=-1 / 2)$ line densities are similar. If real beams behave in this way the fast head tail instability will rarely, if ever, be seen in low energy hadron machines.

\section{REFERENCES}

[1] D. Brandt, G.L. Sabbi, B. Zotter EPAC94 pg 1066 (1994).

[2] A. W. Chao, bf Physics of Collective Beam Instabilities in High Energy Accelerators, Wiley, pg 214, (1993).

[3] see eg. W.H. Press, B.P. Flannery, S.A. Teukolsky, W.T. Vetterling Numerical Recipes, Cambridge University Press, (1986).

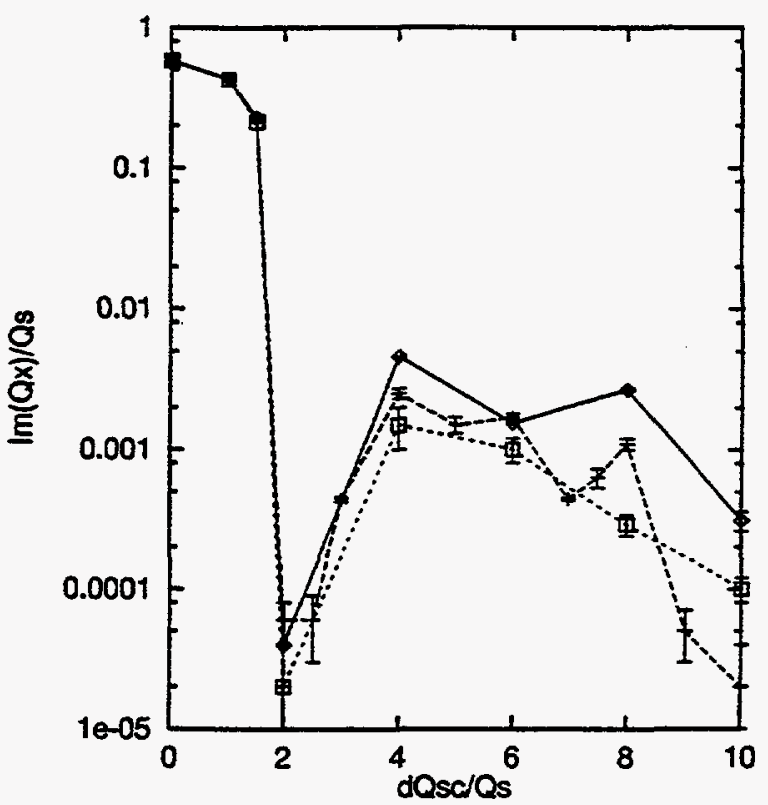

Figure 3: $\operatorname{Im}\left(Q_{x} / Q_{s}\right)$ versus $\Delta Q_{s c} / Q_{s}$ with $\mu=1$ for various values of $n_{\ell}: n_{\ell}=25$, solid line; $n_{\ell}=50$, long dash; $n_{\ell}=100$, short dash. The value of the step function wake was 2.5 times larger than the threshold value with $\Delta Q_{s c}=0$.

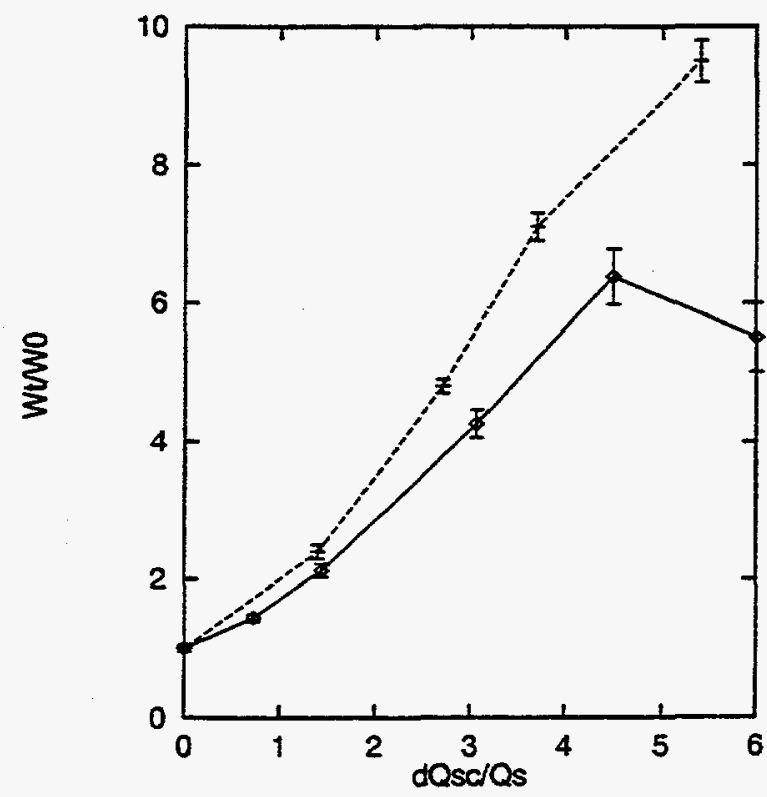

Figure 4: Threshold wake in units of the threshold wake for $\Delta Q_{s c}=0$ versus $\Delta Q_{s c} / Q_{s}$ for different line densities: $\mu=1$, solid line; $\mu=-1 / 2$, long dash. 


\section{DISCLAIMER}

This report was prepared as an account of work sponsored by an agency of the United States Government. Nejther the United States Government nor any agency thereof, nor any of their emplcyees, makes any warranty, express or implied, or assumes any legal liability or responsibility for the accuracy, completeness, or usefulness of any information, apparatus, product, or process disclosed, or represents that its use would not infringe privately owned rights. Reference herein to any specific commercial product, process, or service by trade name, trademark, manufacturer, or otherwise does not necessarily constitute or imply its endorsement, recommendation, or favoring by the United States Government or any agency thereof. The views and opinions of authors expressed herein do not necessarily state or reflect those of the United States Goyernment or any agency thereof. 


\section{DISCLAMMER}

Portions of this document may be illegible in electronic image products. Images are produced from the best available original document. 